\title{
Aumento de la Temperatura de Incubación en Huevos de Ga- llina Araucana (Gallus inauris): Efecto sobre la Mortalidad Embrionaria, Tasa de Eclosión, Peso del Polluelo, Saco Vitelino y de Órganos Internos
}

\author{
Increasing Incubation Temperature of Araucana Hen Eggs (Gallus inauris): Effect \\ on Embryo Mortality, Hatchability, Chick, Yolk Sac and Internal Organs Weight
}

Nancy Ruiz Diaz*; Guillermo Orrego*; Miguel Reyes* \& Mauricio Silva**

RUIZ, D. N.; ORREGO, G.; REYES, M. \& SILVA, M. Aumento de la temperatura de incubación en huevos de gallina Araucana (Gallus inauris): efecto sobre la mortalidad embrionaria, tasa de eclosión, peso del polluelo, saco vitelino y de órganos internos. Int. J. Morphol., 34(1):57-62, 2016.

RESUMEN: Se evaluó el efecto del incremento de la temperatura de incubación en huevos de gallina Araucana sobre la tasa de eclosión, la mortalidad embrionaria, el peso del polluelo, del saco vitelino y de los órganos internos. Los huevos (n=426) fueron distribuidos al azar en: a) grupo Control $(\mathrm{n}=215)$, incubados a $37,5^{\circ} \mathrm{C}$ por 21 días y b) grupo Experimental $(\mathrm{n}=211)$, incubados a $39,5^{\circ} \mathrm{C}$, por 18 días, disminuyéndose a $37,5^{\circ} \mathrm{C}$ los 3 días restantes. En ambos grupos la incubación se realizó con una humedad relativa constante del $55 \%$. La tasa de mortalidad embrionaria total fue significativamente menor ( $\mathrm{P} \leq 0,05)$ para el grupo Control $(14,9$ vs $44,1 \%)$ y consecuentemente la tasa de eclosión fue significativamente mayor $(\mathrm{P} \leq 0,05)$ en este mismo grupo $(97,8$ vs. 80,5 \%). El peso promedio de los polluelos y del saco vitelino a las 36 h post eclosión fue significativamente diferente $(\mathrm{P} \leq 0,05)$ entre ambos grupos $(37,1$ vs. 36,3 y 2,5 vs 6,2 g para el grupo Control y Experimental respectivamente). Por otra parte, el peso de todos los órganos internos fue menor ( $\mathrm{P}$ $\leq 0,05$ ) en polluelos del grupo Experimental. Se concluye que el uso de una temperatura de incubación de $39,5^{\circ} \mathrm{C}$ por 18 días en huevos de gallina araucana influye negativamente, aumentando la mortalidad embrionaria, disminuyendo la tasa de eclosión, el peso del polluelo y de los órganos internos, y aumentando el peso del saco vitelino.

PALABRAS CLAVE: Gallina Araucana; Incubación; Mortalidad embrionaria; Eclosión; Peso polluelo.

\section{INTRODUCCIÓN}

La incubación, natural o artificial, es el proceso por el cual el embrión finaliza su desarrollo morfológico, iniciado dentro de la gallina. Por tanto, la incubación artificial, debe entregar al huevo condiciones ambientales óptimas, similares a las del proceso natural, para el desarrollo embrionario.

El período de incubación del huevo de gallina tiene una duración de 21 días en promedio, 18 de los cuales deben transcurrir en la incubadora y los restantes 3 en la nacedora, a una temperatura $1{ }^{\circ} \mathrm{C}$ menor que durante el proceso previo. La incubación constituye una etapa fundamental de la vida de las aves, ya que durante este periodo se desarrollan y maduran órganos y sistemas fisiológicos, por lo tanto las condiciones ambientales existentes durante el desarrollo embrionario serán determinantes para el creci- miento y desarrollo del polluelo, pudiendo también influir en el rendimiento productivo y la salud en la edad adulta (Fleming et al., 2004; Molenaar et al., 2010).

La duración y sobre todo los resultados de la incubación, dependen de un conjunto de factores, entre los que se pueden destacar el periodo de almacenamiento previo de los huevos, las condiciones ambientales de pre incubación, el tamaño del huevo, el grosor y porosidad de la cáscara (Gonzáles et al., 2003), así como la temperatura de incubación, humedad relativa, contenido de oxígeno y anhídrido carbónico del aire, presión barométrica y frecuencia de volteo de los huevos durante la incubación (Givisiez et al., 2003). Dentro de estos factores la temperatura de incubación es una variable crucial (Decuypere \& Michels, 1992; Reijrink et al., 2009; Molenaar

\footnotetext{
* Escuela de Medicina Veterinaria, Universidad Santo Tomás, Sede Temuco, Chile.

** Escuela de Medicina Veterinaria, Universidad Católica de Temuco, Temuco, Chile.
} 
et al.), siendo la temperatura óptima aquella que permite lograr la máxima tasa de eclosión y calidad de polluelos (Decuypere \& Michels; French, 1997). Los rangos descritos de temperaturas óptimas de incubación para los embriones de aves varían entre 37 y $37,5{ }^{\circ} \mathrm{C}$ (Decuypere \& Michels), 37,5 y $37,8{ }^{\circ} \mathrm{C}$ (Tullet, 1990) y 35 hasta $40,5^{\circ} \mathrm{C}$ (French). Cualquier cambio en la temperatura durante la incubación puede afectar el tamaño del embrión, el crecimiento de sus órganos, la tasa metabólica, el desarrollo fisiológico y el éxito de la eclosión (Yalçin \& Siegel, 2003).

Los efectos observados del aumento de temperatura sobre la incubabilidad de los huevos son muchas veces contradictorios (Oviedo-Rondon et al., 2010). Esto podría deberse a diferencias en las características propias del huevo, como el grosor y porosidad de la cáscara de las diferentes líneas genéticas (Bennett, 1992; Roque \& Soares, 1994; Gualhanone et al., 2012), lo que podría influir sobre la conductibilidad del calor y la deshidratación del huevo en el proceso de incubación. Las características estructurales de la cáscara del huevo influyen en la habilidad que tendría el vapor de agua, el $\mathrm{O}_{2}$ y el $\mathrm{CO}_{2}$ para atravesar los poros hacia y/o desde el embrión en desarrollo (Bennett; Roque \& Soares; Liao et al., 2013), presentando huevos de cáscara delgada mayor propensión a la deshidratación del embrión durante la incubación (Wilson, 1997).

La gallina Araucana (Gallus inauris) es un ave distribuida ampliamente en el sur de Chile, que posee un potencial productivo-comercial debido a la postura de huevos verde/ azulados. El huevo de la gallina Araucana posee una cáscara de mayor grosor $(0,06 \mathrm{~mm})$ y resistencia $\left(3,1 \mathrm{Kg} / \mathrm{cm}^{2}\right)$ que la de las líneas genética de gallinas comerciales (Rodríguez Ríos et al., 2014), lo cual se debería a que presenta un $7 \%$ más de calcio (Simmons \& Somes, 1985). Se ha descrito en broilers que huevos de cáscara delgada presentan una incubabilidad 3$9 \%$ menor que huevos de cáscara de mayor grosor (Bennett), con variaciones significativas en los porcentajes de mortalidad embrionaria observados entre huevos de distinto grosor de cáscara (Roque \& Soares, 1994; O’Dea et al., 2004).

El objetivo de este trabajo fue evaluar el efecto del incremento de la temperatura de incubación en huevos de gallina Araucana sobre la tasa de eclosión, la mortalidad embrionaria, el peso del polluelo, del saco vitelino y de los órganos internos.

\section{MATERIAL Y MÉTODO}

El presente estudio fue realizado en las dependencias de la Escuela de Medicina Veterinaria de la Universidad Santo Tomás, sede Temuco, entre los meses de agosto y septiembre de 2013.
Huevos verde-azulados embrionados de gallina Araucana fueron obtenidos de criaderos de pequeños productores locales, ubicados en la Provincia de Cautín, Región de la Araucanía, Chile. Los huevos fueron colectados el mismo día de la postura y trasladados a una sala de almacenamiento con una temperatura de $10^{\circ} \mathrm{C}$ y una humedad relativa de $65 \%$, en donde permanecieron por 5 días. Luego se realizó la selección de los huevos en base a criterios de peso, forma, limpieza e integridad de cáscara, y a su posterior desinfección.

Los huevos verde-azulados seleccionados $(n=426)$ fueron distribuidos al azar en los siguientes grupos: a) Control, $(\mathrm{n}=215)$ huevos incubados a $37,5^{\circ} \mathrm{C}$ en forma constante por un periodo de 21 días y b) Experimental, $(n=211)$ huevos incubados por un periodo de 21 días, a $39,5^{\circ} \mathrm{C}$ durante los primeros 18 días, disminuyéndose a $37,5^{\circ} \mathrm{C}$ los últimos 3 días. En ambos grupos los huevos fueron incubados con una humedad relativa constante de $55 \%$. Se realizaron 4 repeticiones del proceso de incubación (52-54 huevos/grupo/repetición) por la capacidad de la incubadora/ nacedora (Thermal Hova Bator, modelo 1602-N, G.Q.F. Manufacturing, Estados Unidos).

Las variables evaluadas fueron mortalidad embrionaria a los 7, 14 y 19 días de incubación, tasa de eclosión, peso del polluelo, peso del saco vitelino y de los órganos internos (corazón, hígado, molleja, proventrículo e intestino delgado) a las $36 \mathrm{~h}$ post eclosión. La muerte embrionaria fue determinada mediante la observación directa de ausencia de signos de viabilidad (vascularización, visualización del ojo y movilidad embrionaria) utilizando un ovoscopio portátil (Olba B.V., Super flash SL-SF, Holanda). Adicionalmente, el diagnóstico de muerte embrionaria fue corroborado a través de la extracción de los embriones y la visualización directa de los mismos. La tasa de eclosión se calculó considerando la mortalidad acumulada al final de la incubación. El pesaje de todos los polluelos nacidos fue realizado a las $36 \mathrm{~h}$ post eclosión, utilizando una balanza digital de precisión. Posteriormente al pesaje se procedió a la eutanasia de los polluelos, mediante embolia gaseosa de acuerdo a lo estipulado en el Consejo Canadiense en cuidados de animales de experimentación (CCAC), para luego extraer y pesar el saco vitelino y los órganos internos utilizando una balanza analítica de precisión (Radwag, AS22OC2, Polonia).

Los porcentajes de mortalidad embrionaria al día 7 , 14 y 19 y tasa de eclosión fueron comparados mediante la prueba de Chi cuadrado. El peso del polluelo, saco vitelino y los órganos internos fue analizado mediante la prueba de $t$ de Student. Los análisis fueron realizados utilizando el programa estadístico GraphPrism ${ }^{\circledR}$ versión 5.0. El nivel de significancia establecido fue de $\mathrm{P} \leq 0,05$. 


\section{RESULTADOS}

La mortalidad embrionaria observada en la etapa inicial (Día 7) e intermedia (Día 14) del período de incubación, así como la tasa total fue superior $(\mathrm{P} \leq 0,05)$ en el grupo Experimental (Tabla I).

La tasa de eclosión fue significativamente afectada por el incremento de la temperatura, siendo superior ( $\mathrm{P}$ $\leq 0,05$ ) en el grupo Control (Fig. 1).

El peso del polluelo y los órganos internos (corazón, hígado, molleja, proventrículo e intestino delgado) fueron inferiores $(\mathrm{P} \leq 0,05)$ en el grupo Experimental con respecto al grupo Control (Tabla II). Por el contrario, el peso del saco vitelino presentó un menor $(\mathrm{P} \leq 0,05)$ peso en el Grupo Con- trol (2,5 g) en comparación al Grupo Experimental (6,2 g; Tabla II), lo que refleja una menor capacidad de absorción del vitelo por parte de los polluelos de este último grupo.

\section{DISCUSIÓN}

El presente estudio demostró que para huevos de gallina Araucana (Gallus inauris) el incremento en $2{ }^{\circ} \mathrm{C}$ de la temperatura, durante los primeros 18 días de la incubación, afectó negativamente la viabilidad de los polluelos, lo que se reflejó en un aumento de la tasa de mortalidad embrionaria y una disminución de la tasa de eclosión. Por otra parte redujo el peso de los polluelos nacidos y el de sus órganos internos, al igual que la capacidad de absorción del vitelo por parte de los polluelos.

Tabla I. Mortalidad embrionaria (porcentaje) durante la incubación de huevos de gallina araucana a $37,5^{\circ} \mathrm{C}$ (grupo Control) y $39,5^{\circ} \mathrm{C}$ (grupo Experimental) por un periodo de 18 días.

\begin{tabular}{lclll}
\hline \multirow{2}{*}{ Grupo } & \multicolumn{4}{c}{ Mortalidad embrionaria (\%) } \\
& Día 7 & \multicolumn{1}{c}{ Día 14 } & \multicolumn{1}{c}{ Día 18 } & Total \\
\hline Control & $2,3 \mathrm{a}(5 / 215)$ & $9^{\mathrm{a}}(19 / 210)$ & $4,28 / 191$ & $14,9^{\mathrm{a}} 32 / 215$ \\
Experimental & $9 \mathrm{~b}(19 / 211)$ & $36,5^{\mathrm{b}}(70 / 192)$ & $3,3(4 / 122)$ & $44,1^{\mathrm{b}}(93 / 211)$ \\
\hline a,b Proporciones con superíndices distintos difieren estadísticamente $(\mathrm{P}<0,05)$
\end{tabular}

a,b Proporciones con superíndices distintos difieren estadísticamente $(\mathrm{P} \leq 0,05)$

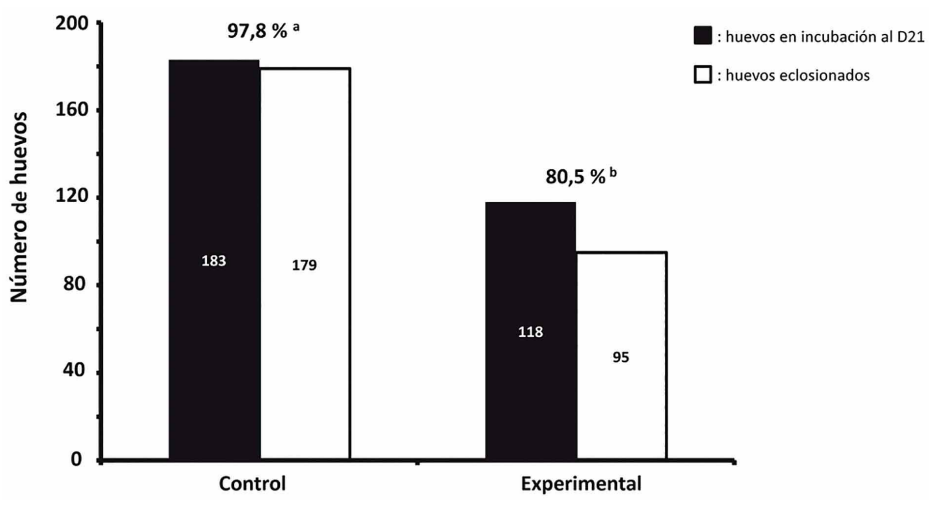

Fig. 1. Tasa de eclosión en huevos de gallina araucana incubados a $37,5^{\circ} \mathrm{C}$ (grupo Control) y $39,5^{\circ} \mathrm{C}$ (grupo Experimental) por un periodo de 18 días. a,b Porcentajes con superíndices distintos difieren estadísticamente $(\mathrm{P} \leq 0.05)$.

Tabla II. Peso (promedio \pm DE) de polluelo, saco vitelino y órganos internos de polluelos de gallina araucana a las 36 horas pos eclosión, incubados a $37,5{ }^{\circ} \mathrm{C}$ (grupo Control) y $39,5^{\circ} \mathrm{C}$ (grupo Experimental) por un periodo de 18 días.

\begin{tabular}{|c|c|c|c|c|c|c|c|}
\hline \multirow[b]{2}{*}{ Grupos } & \multicolumn{7}{|c|}{ Peso a las 36 horas pos eclosión (g) } \\
\hline & Polluelo & $\begin{array}{c}\text { Saco } \\
\text { vitelino }\end{array}$ & Corazón & Hígado & Proventrículo & Molleja & $\begin{array}{r}\text { Intestino } \\
\text { delgado }\end{array}$ \\
\hline $\begin{array}{l}\text { Control } \\
(n=179)\end{array}$ & $37,1 \pm 0,1$ a & $2,5 \pm 0,9^{\text {a }}$ & $0,5 \pm 0,5$ a & $1,5 \pm 0,1^{\mathrm{a}}$ & $0,5 \pm 0,1$ a & $1,5 \pm 0,2^{\mathrm{a}}$ & $1,4 \pm 0,1^{a}$ \\
\hline $\begin{array}{l}\text { Experimental } \\
(n=95)\end{array}$ & $36,3 \pm 0,1$ b & $6,2 \pm 1,6^{\mathrm{b}}$ & $0,2 \pm 0,1^{b}$ & $1,0 \pm 0,1^{\mathrm{b}}$ & $0,4 \pm 0,2$ ь & $1,2 \pm 0,2^{\mathrm{b}}$ & $1,2 \pm 0,1 \mathrm{~b}$ \\
\hline
\end{tabular}

a,b Proporciones con superíndices distintos difieren estadísticamente $(\mathrm{P} \leq 0,05)$. 
Lo anterior sugiere que a pesar de que los huevos de gallina Araucana presentan un mayor grosor de la cáscara, lo cual reduce las posibilidades de deshidratación del embrión durante la incubación (Wilson), favoreciendo su sobrevivencia, el incremento de la temperatura de incubación tuvo un efecto negativo sobre la viabilidad de los embriones/polluelos, similar a lo observado en gallinas de líneas comerciales (Roque \& Soares; O’Dea et al.). La relación entre este efecto negativo y la magnitud del incremento de la temperatura $\left(2^{\circ} \mathrm{C}\right)$, o el período de tiempo utilizado $(18$ días), requiere de mayores investigaciones.

Por otra parte, nuestros resultados confirman lo reportado previamente por otros autores quienes observaron efectos negativos del aumento sostenido de la temperatura de incubación sobre la sobrevivencia embrionaria (Lourens et al., 2005; Ipek \& Sahan, 2006) en gallinas de líneas comerciales, los cuales se producen debido a la hipertermia del embrión. Sin embargo, la exposición a temperaturas de incubación elevadas $\left(38,5^{\circ} \mathrm{C}\right.$ ) por periodos cortos de tiempo ( $4 \mathrm{a} 6 \mathrm{~h}$ ) durante los días 14 y 16 de incubación no produjeron incrementos de la tasa de mortalidad embrionaria (Askit et al., 2010).

Producto del incremento de la temperatura se reportan aumentos de la mortalidad embrionaria en las fases intermedia y tardía de incubación (Lourens et al., 2005; Ipek \& Sahan, 2006; Hulet, 2007). En el presente estudio los incrementos en la tasa de mortalidad embrionaria fueron observados también en la etapa intermedia (Día 14) de la incubación; sin embargo, incrementos significativos también fueron observados en la etapa inicial (Día 7), los cuales pudieron deberse a la utilización de una temperatura mayor de incubación $\left(39,5^{\circ} \mathrm{C}\right)$ en comparación a la utilizada en el estudio de los autores previamente indicados $\left(38,5^{\circ} \mathrm{C}\right)$.

Conjuntamente, se ha reportado que el incremento de la temperatura de incubación provoca una disminución de la capacidad de eclosión (Lourens et al., 2005; Collin et al., 2007). Aumentos de $1{ }^{\circ} \mathrm{C}$ por sobre la temperatura óptima $\left(37,8^{\circ} \mathrm{C}\right)$ a partir del día 13 de incubación provocan una reducción significativa de la tasa de eclosión de los huevos de reproductoras de broiler (Givisiez et al.). De la misma manera, en huevos de gallina Araucana, el incremento de la temperatura de incubación a una temperatura constante de $39,5^{\circ} \mathrm{C}$ por 18 días, indujo una tasa de mortalidad embrionaria acumulada significativamente mayor que una temperatura de 37,5 ${ }^{\circ} \mathrm{C}$, disminuyendo consecuentemente la tasa de eclosión, la cual experimentó una reducción $>15 \%$.

Tasas de desarrollo menor y consecuentemente la reducción del peso al nacimiento de polluelos expuestos a un aumento de temperatura de incubación han sido descritos en líneas comerciales de gallinas (Yahav et al., 2004;
Leksrisompong et al., 2007). Los resultados del presente estudio son coincidentes. La razón de esta disminución de peso se debería a una menor eficiencia de la utilización de proteínas para el crecimiento, así como a una menor eficiencia del metabolismo energético del polluelo, ya que la temperatura de incubación determina la tasa de absorción de la yema y la utilización de la albúmina por parte del embrión, y por tanto el desarrollo del mismo durante el incubación (Lourens et al., 2007; O`Dea et al.). Adicionalmente, Ipek \& Sahan indican que una temperatura más alta en el interior de los huevos podría aumentar el gradiente de temperatura entre el cuerpo del embrión y la incubadora, que a su vez genera mayor disipación de calor por evaporación y por lo tanto una pérdida de peso mayor antes de la eclosión del polluelo.

Por otra parte, la disminución de peso del embrión se ha vinculado al aumento del tamaño del saco vitelino en polluelos (Hulet). Coincidentemente en nuestro estudio el aumento de la temperatura de incubación implicó un aumento del peso del saco vitelino. Estos resultados coinciden con lo señalado por diversos autores (Lourens et al., 2007; Leksrisompong et al.) quienes observaron que las temperaturas superiores a 38,5 ${ }^{\circ} \mathrm{C}$ influyen en el desarrollo del polluelo produciendo una menor masa corporal y un mayor tamaño del saco vitelino. Según lo descrito por autores como Wineland et al. (2000) y Lourens et al. (2007) temperaturas de incubación, por sobre el óptimo, alterarían la capacidad de utilización de los nutrientes presentes en el saco vitelino por parte de los polluelos $\left(37,5^{\circ} \mathrm{C}\right)$.

Coincidentemente con la disminución del peso del polluelo se observó la disminución del peso de los órganos internos, producto del incremento de la temperatura de incubación. Nuestros resultados coinciden con los reportados por múltiples autores (Wineland et al.; Leksrisompong et al.) quienes observaron la reducción del peso del intestino delgado, molleja, proventrículo e hígado en rangos de 13 a $16 \%$. Estos autores sugieren que la causa de esta disminución de peso se debería a la reducción de la masa de tejidos y a la reducción de la actividad enzimática. A su vez, la disminución observada en el peso del corazón coincide con las observaciones de Molenaar et al. quienes reportaron que este órgano presenta menor tamaño y peso así como cambios en su morfología, principalmente en la proporción del ventrículo izquierdo y el derecho, como consecuencia de una temperatura de incubación elevada $\left(38,8^{\circ} \mathrm{C}\right)$.

Podemos concluir entonces, que en huevos de gallina Araucana (Gallus inauris) el incremento de la temperatura en $2{ }^{\circ} \mathrm{C}$, durante los primeros 18 días de la incubación, incrementa la tasa de mortalidad embrionaria, disminuye la tasa de eclosión, el peso de los polluelos y de sus órganos internos, reduciéndose a la vez la absorción del vitelo por parte del polluelo 
RUIZ, D. N.; ORREGO, G.; REYES, M. \& SILVA, M. Aumento de la temperatura de incubación en huevos de gallina Araucana (Gallus inauris): efecto sobre la mortalidad embrionaria, tasa de eclosión, peso del polluelo, saco vitelino y de órganos internos. Int. J. Morphol., 34(1):57-62, 2016.

RUIZ, D. N.; ORREGO, G.; REYES, M. \& SILVA, M. Increasing incubation temperature of Araucana hen eggs (Gallus inauris): effect on embryo mortality, hatchability, chick, yolk sac and internal organs weight. Int. J. Morphol., 34(1):57-62, 2016.

SUMMARY: The aim of this study was to evaluate the effect of increasing incubation temperature of Araucana hen eggs on embryo mortality, hatchability,chick, yolk sac and internal organs weight. Eggs $(n=426)$ were randomly distributed into: a) Control group $(n=215)$, which were incubated at $37.5^{\circ} \mathrm{C}$ for 21 days or b) Experimental group $(n=211)$, which were incubated at $39.5^{\circ} \mathrm{C}$, for 18 days and then at $37.5{ }^{\circ} \mathrm{C}$ for the remaining 3 days. In both groups eggs were incubated with a relative humidity of $55 \%$. The embryo mortality rate was greater $(\mathrm{P} \leq 0.05)$ for the experimental than the control group ( $44.1 \mathrm{vs.} 14.9 \%$ respectively). On the contrary, hatching rate was greater $(\mathrm{P} \leq 0.05)$ for the control than the experimental group ( 97.8 vs. $80.5 \%$ respectively). The average weight of chicks, at 36 $\mathrm{h}$ of age, was lower $(\mathrm{P} \leq 0.05)$ for the experimental than control group (36.3 vs. $37.01 \mathrm{~g}$ respectively). Also, increasing incubation temperature reduced $(\mathrm{P} \leq 0.05)$ internal organs weight of chicks in the Experimental compared to Control group. On the other hand, yolk sac weight was reduced ( $\mathrm{P}<0.05 ; 2.5$ vs. $6.2 \mathrm{~g}$ for Control and Experimental group respectively). We conclude that increasing the incubation temperature of Araucana hen eggs up to $39.5^{\circ} \mathrm{C}$ for 18 days has a negative effect on embryo survival, hatchability, chick and internal organs weight and absorption of yolk.

KEY WORDS: Araucana hen; Incubation; Embryo mortality; Hatchability; Chick weight.

\section{REFERENCIAS BIBLIOGRÁFICAS}

Aksit, M.; Yalçin, S.; Yenisey, C. \& Ozdemir, D. Brooding temperatures for chicks acclimated to heat during incubation: effects on post-hatch intestinal development and body weight under heat stress. Br. Poult. Sci., 51(3):444-52, 2010.

Bennett, C. D. The influence of shell thickness on hatchability in commercial broiler breeder flocks. J. Appl. Poult. Res., $1(1): 61-5,1992$.

Collin, A; Berri, C.; Tesseraud, S.; Rodón, F. E.; Skiba-Cassy, S.; Crochet, S.; Duclos, M. J.; Rideau, N.; Tona, K.; Buyse, J.; Bruggeman, V.; Decuypere, E.; Picard, M. \& Yahav, S. Effects of thermal manipulation during early and late embryogenesis on thermotolerance and breast muscle characteristics in broiler chickens. Poult. Sci., 86(5):795-800, 2007.

Decuypere, E. \& Michels, H. Incubation temperature as a management tool: A review. World's Poult. Sci. J., 48:28-38, 1992.

Fleming, T. P.; Kwong, W. Y.; Porter, R.; Ursell, E.; Fesenko, I.; Wilkins, A.; Miller, D. J.; Watkins, A. J. \& Eckert, J. J. The embryo and its future. Biol. Reprod., 71(4):1046-54, 2004.

French, N. A. Modeling incubation temperature: the effects of incubator design, embryonic development, and egg size. Poult. Sci., 76(1):124-33,1997.

Givisiez, P. E. N.; Furlan, R. L.; Malheiros, E. B. \& Macari, M. Incubation and rearing temperature effects on Hsp70 levels and heat stress response in broilers. Can. J. Anim. Sci., 83(2):213-20, 2003.

Gonzales, E.; Kondo, N.; Saldanha, E. S.; Loddy, M. M.; Careghi, C. \& Decuypere, E. Performance and physiological parameters of broiler chickens subjected to fasting on the neonatal period. Poult. Sci., 82(8):1250-6, 2003.
Gualhanone, A.; Furlan, R. L.; Fernandez-Alarcon, M. F. \& Macari, M. Effect of breeder age on eggshell thickness, surface temperature, hatchability and chick weigh. Rev. Bras. Cienc. Avic., 14(1):9-14, 2012.

Hulet, R. M. Managing incubation: where are we and why? Poult. Sci., 86(5):1017-9, 2007.

Ipek, A. \& Sahan, U. Effects of cold stress on broiler performance and ascites susceptibility. Asian Australas. J. Anim. Sci., 19(5):734-8, 2006.

Leksrisompong, N.; Romero-Sanchez, H.; Plumstead, P. W:; Brannan, K. E. \& Brake, J. Broiler incubation. 1. Effect of elevated temperature during late incubation on body weight and organs of chicks. Poult. Sci., 86(12):2685-91, 2007.

Liao, B.; Qiao, H. G.; Zhao, X. Y.; Bao, M.; Liu, L.; Zheng, C. W.; Li, C. F. \& Ning, Z. H. Influence of eggshell ultrastructural organization on hatchability. Poult. Sci., 92(8):2236-9, 2013.

Lourens, A.; van den Brand, H.; Meijerhof, R. \& Kemp, B. Effect of eggshell temperature during incubation on embryo development, hatchability, and posthatch development. Poult. Sci., 84(6):914-20, 2005.

Lourens, A.; van den Brand, H.; Heetkamp, M. J.; Meijerhof, R. \& Kemp, B. Effects of eggshell temperature and oxygen concentration on embryo growth and metabolism during incubation. Poult. Sci., 86(10):2194-9, 2007.

Molenaar, R.; Meijerhof, R.; van den Anker, I.; Heetkamp, M. J.; van den Borne, J. J.; Kemp, B. \& van den Brand, H. Effect of eggshell temperature and oxygen concentration on survival rate and nutrient utilization in chicken embryos. Poult. Sci., 89(9):2010-21, 2010. 
RUIZ, D. N.; ORREGO, G.; REYES, M. \& SILVA, M. Aumento de la temperatura de incubación en huevos de gallina Araucana (Gallus inauris): efecto sobre la mortalidad embrionaria, tasa de eclosión, peso del polluelo, saco vitelino y de órganos internos. Int. J. Morphol., 34(1):57-62, 2016.

O’Dea, E. E.; Fasenko, G. M.; Feddes, J. J.; Robinson, F. E.; Segura, J. C.; Ouellette, C. A. \& van Middelkoop, J. H. Investigating the eggshell conductance and embryonic metabolism of modern and unselected domestic avian genetic strains at two flock ages. Poult. Sci., 83(12):2059-70, 2004.

Oviedo-Rondón, E. O.; Hume, M. E.; Barbosa, N. A.; Sakomura, N. K.; Weber, G. \& Wilson, J. W. Ileal and cecal microbial populations in broilers given specific essential oil blends and probiotics in two consecutive grow-outs. Avian Biol. Res., 3(4):157-69, 2010.

Reijrink, I. A.; Meijerhof, R.; Kemp, B.; Graat, E. A. \& van den Brand, H. Influence of prestorage incubation on embryonic development, hatchability, and chick quality. Poult. Sci., 88(12):2649-60, 2009.

Rodríguez Ríos, H.; Abud, G.; Figueroa, M.; Tima Péndola, M. \& Campos Parra, J. Evaluación y características de la gallina Araucana (Gallus inauris Castelloi) como ave de postura. Chil. J. Agric. Anim. Sci., 30(2):139-46, 2014.

Roque, L. \& Soares, M. C. Effects of eggshell quality and broiler breeder age on hatchability. Poult. Sci., 73(12):1838-45, 1994.

Simmons III, R. W. \& Somes Jr., R. G. Chemical characteristics of araucana chicken eggs. Poult. Sci., 64(7):1264-8, 1985.

Tullet, S. G. Science and the art of incubation. Poult. Sci., 69(1):115,1990 .

Wilson, H. R. Effects of maternal nutrition on hatchability. Poult. Sci., 76(1):134-43, 1997.

Wineland, M. J.; Mann, K. M.; Fairchild, B. D. \& Christensen, V. L. Effect of different setter and hatcher temperatures upon the broiler embryo. Poult. Sci., 79(Suppl. 1):181, 2000.

Yalçin, S. \& Siegel, P. B. Developmental stability of broiler embryos in relation to length of egg storage prior to incubation. J. Poult. Sci., 40(4):298-308, 2003.

Yahav, S.; Collin, A.; Shinder, D. \& Picard, M. Thermal manipulations during broiler chick embryogenesis: effects of timing and temperature. Poult. Sci., 83(12):1959-63, 2004.

\section{Dirección para Correspondencia: Mauricio Silva \\ Escuela de Medicina Veterinaria Universidad Católica de Temuco \\ Temuco \\ CHILE}

Email: masilva@uct.cl

Recibido : 28-07-2015

Aceptado: 19-11-2015 